Commentary/Glover: Separate visual representations in the planning and control of action

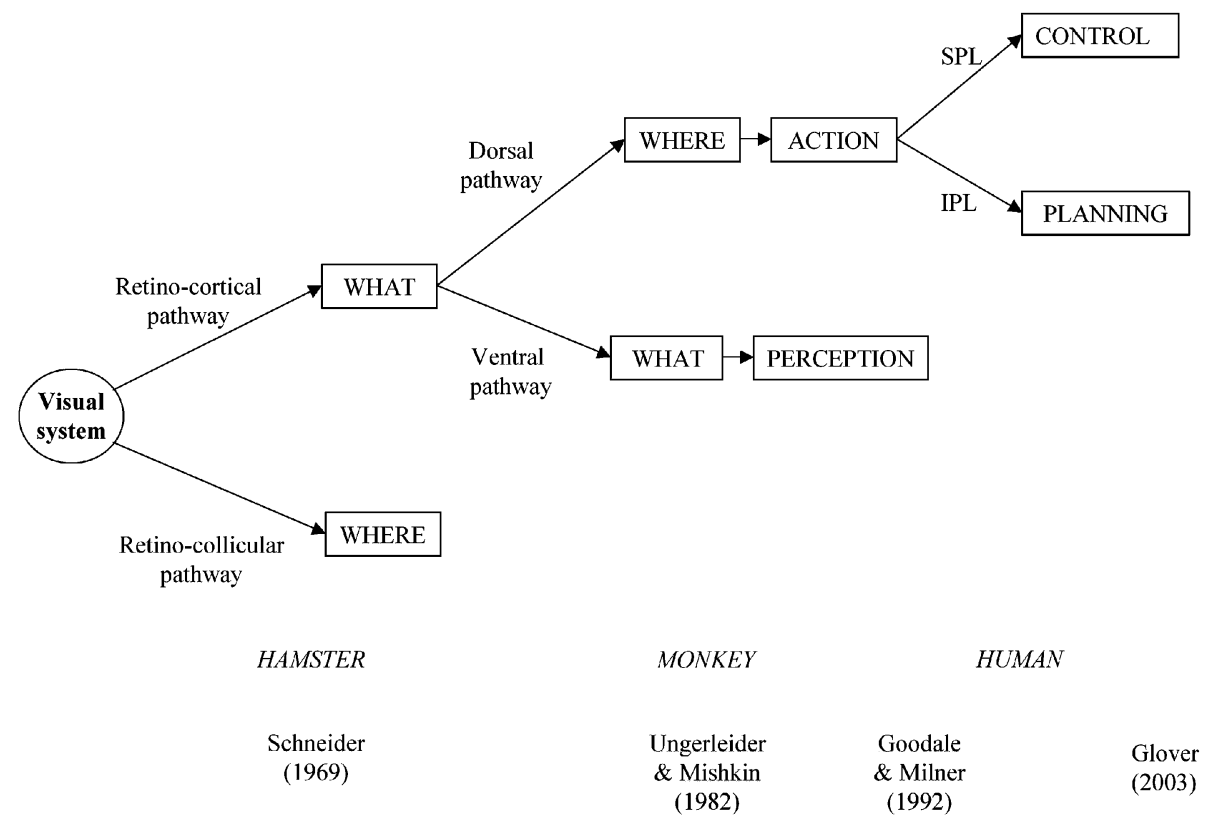

Figure 1 (Latto). The binary cascade model of the development of our understanding of the organisation of the visual system.

3. The fact that English has a specific part of speech, the comparative inflection of the adjective, to identify a binary category (higher, lower, etc.) raises the possibility that the tendency to form binary subdivisions is a fundamental process in human thought. Maybe, as with some primitive counting systems ("one, two, many"), two subdivisions are as many specific categories as we can easily cope with.

\section{Automaticity and inhibition in action planning}

\author{
Matthew R. Longo and Bennett I. Bertenthal \\ Department of Psychology, University of Chicago, Chicago, IL 60637. \\ mlongo@uchicago.edubertenthal@uchicago.edu \\ http://home.uchicago.edu/ mlongo \\ www.ccp.uchicago.edu/ bbertent
}

Abstract: We question the generalizability of Glover's model because it fails to distinguish between different forms of planning. The highly controlled experimental situations on which this model is based, do not reflect some important factors that contribute to planning. We discuss several classes of action that seem to imply distinct planning mechanisms, questioning Glover's postulation of a single "planning system."

Glover characterizes planning as a discrete and monolithic system operating, to a large extent, prior to the initiation of an action. While this model is a welcome addition to recent models distinguishing perception and action, we believe that the mechanisms underlying planning may be much more diverse than suggested by the target article. Humans are unquestionably adept at planning skillful action, yet this function need not be meditated by a single system any more than memory, say, need consist of a single mechanism. The mechanisms underlying planning in the highly controlled experimental situations cited in support of the planning-control model do not do justice to the full diversity and complexity of human action. It has been noted, for example, that apraxic patients demonstrate strikingly few difficulties interacting with objects in naturalistic situations even as they fail completely to demonstrate such ability in controlled laboratory situations (see Cubelli \& Della Sala 1996). It is important to further differentiate the construct of planning if the planning-control model is to generalize beyond simple actions involving pointing, reaching, and grasping at isolated objects.
One important distinction made by researchers in diverse fields is that between direct and mediated actions. The former category assumes a direct mapping between perception and action, whereas the latter assumes that action is mediated by stored representations. Glover emphasizes the role of mediated actions, focusing on the relation of so-called visuokinesthetic engrams in the inferior parietal lobe (IPL) to planning (sect. 4.1.1, para. 4). In addition to the evidence he cites, such representational mediation is implied by patients with visuo-imitative apraxia who are able to imitate familiar but not unfamiliar gestures (Goldenberg \& Hagmann 1997). Patients with so-called pantomime agnosia, on the other hand, are able to imitate gestures that they are unable to recognize (Rothi et al. 1986), implying a separate pathway from perception to action that bypasses stored representations used by patients with visuo-imitative apraxia. To account for such dissociations, recent models of deficits in apraxia (e.g., Cubelli et al. 2000; Rothi et al. 1991) have found it necessary to include multiple pathways from perception to action. There is no place for a single "planning system" in these models. Rather, planning is conceptualized as consisting of multiple and diverse neural circuits that differentially contribute as a function of task, context, experience, and the available control and coordination of the relevant actions.

Some researchers have also distinguished between actions that are externally versus internally motivated, the former made in response to some distal cue, the latter generated volitionally by the subject on the basis of stored representations. These two types of behavior are mediated largely by distinct lateral and medial premotor systems respectively (Goldberg 1985; 1987; Passingham 1993).

A related distinction is that between automatic and volitional actions. In some cases, perceptual stimuli seem to elicit corresponding action plans automatically. Rizzolatti and colleagues, for example, have identified neurons in the monkey's premotor cortex that code goal-directed actions and are also activated visually by objects affording that action ("canonical neurons"; Rizzolatti et al. 1988) or by the same action being performed by the experimenter ("mirror neurons"; di Pellegrino et al. 1992). Recent behavioral studies in humans have demonstrated similar effects. Gentilucci (2002), for example, has demonstrated that the affordances of task-irrelevant objects systematically influence reach 
kinematics. Subjects' grip aperture is increased when reaching for a dowel sitting on a larger as opposed to a smaller sphere, even though the target dowel does not differ between conditions. Similarly, when attention is diverted, individuals have been observed to unwittingly engage in behavioral mimicry (Chartrand \& Bargh 1999; Stengel 1947). Hommel (2000) reviews evidence of at least four distinct forms of automatic stimulus response translation which appear to arise from different processes.

Such automatic activation is most apparent when inhibitory control is lacking, either following brain insult or during early infancy. Denny-Brown (1958), for example, observed that following lesions of the medial frontal lobes, patients showed a compulsive drive to interact with objects in their environment, what he termed magnetic apraxia. Similar compulsivity was observed by Lhermitte (1983; Lhermitte et al. 1986) following frontal lobe damage. These patients demonstrated a total dependence on environmental stimuli to guide their actions, compulsively using objects (utilization behaviors) and mimicking the experimenter (imitation behavior). Similar behaviors can be observed in infancy. Baldwin (1892) described the young infant as "suggestible," in that environmental stimuli would automatically evoke congruent behavior. Such behaviors were observed by McGraw (1941), who found early reaching movements in response to objects notable for their "autonomous quality," in that these reactions seemed "in no way connected with a desire on the part of the child to possess or manipulate the object" (p. 130).

Our own work (Longo 2003) demonstrates that perseverative search can be elicited in nine-month-old infants to a location where they had seen the experimenter reach is but had not reached to themselves, suggesting that the perception of the experimenter's action had activated analogous motor responses in the infant. This is consonant, as well, with findings of imitation in very young infants (e.g., Meltzoff \& Moore 1977), which can also be explained in terms of motor priming (Kinsbourne 2002), followed by a decline over the first few months of life (Fontaine 1984; Maratos 1982), presumably on account of increased inhibitory control.

Glover describes planning as having "the goal of selecting and initiating an adaptive motor program" (sect. 1.1.2, para. 1). In complex ecological situations, however, planning frequently has the goal not only of selecting adaptive motor programs, but also inhibiting non-adaptive motor programs which have been automatically activated by environmental stimuli. Consider, for example, the mostly automated actions of a skilled baseball player hitting a pitched ball, relative to the tentative and uncoordinated actions of a novice. The former player not only does a better job of anticipating and coordinating his or her movements, but also is more adept at inhibiting erroneous movements, such as swinging at balls out of the strike zone. Such inhibitory control does not fit easily into the planning-control framework articulated by Glover and, therefore, we question the sufficiency of the current model to account for much of naturalistic behavior.

\section{ACKNOWLEDGMENT}

Matthew R. Longo was supported by a fellowship from the National Science Foundation during the preparation of this commentary.

\section{Evidence from optic ataxia does not support a distinction between planning and control mechanisms in human motor control}

\author{
Roger Newport, Sally Pears, and Stephen R. Jackson \\ School of Psychology, University of Nottingham, University Park, Nottingham, \\ NG13 8DB, United Kingdom. roger.newport@nottingham.ac.uk \\ IpxsIp@nottingham.ac.uk stephen.jackson@nottingham.ac.uk \\ http://www.psychology.nottingham.ac.uk/staff/rwn/ \\ http://www.psychology.nottingham.ac.uk/staff//pxs/p/ \\ http://www.psychology.nottingham.ac.uk/staff/srj/
}

Abstract: Evidence from optic ataxic patients with bilateral lesions to the superior parietal lobes does not support the view that there are separate planning and control mechanisms located in the IPL and SPL respectively. The aberrant reaches of patients with bilateral SPL damage towards extrafoveal targets seem to suggest a deficit in the selection of appropriate motor programmes rather than a deficit restricted to on-line control.

Glover is to be commended for his extensive review of this largely unresolved current issue in motor control. His planning-control model, however, is not as parsimonious as many existing models and is unconvincing for several reasons. Furthermore, the distinction between planning and control processes appears rather arbitrary and unnecessary when a single continuous mechanism for both would be far more practical and elegant. Glover's distinction between planning and control mechanisms is an expansion of traditional models based upon the observation that on-line adjustments are usually seen only towards the latter stages of fast aiming movements. The lateness of such adjustments, however, probably has as much to do with the inertial properties of the limb as they have with a putative switch between planning and control mechanisms. Although the model might successfully explain experimental data more accurately than the perception and action model (Milner \& Goodale 1995), a more succinct and parsimonious model is the inverse-forward model (Wolpert \& Ghahramani 2000). The latter not only allows for a single continuous recursive process active from before movement onset until movement endpoint but can also be applied to eye movements, whereas the planning-control model cannot. Overall, the planning-control model does not advance our understanding of motor control above and beyond existing models; although we believe the model has numerous flaws, this commentary will focus specifically on optic ataxia and deficits of on-line control.

According to the planning-control model, a patient suffering from optic ataxia has deficits that are limited to on-line control but has unimpaired movement planning. The "planning" stage has access to all of the necessary spatial characteristics relating to the actor, effector, and target in order to plan a goal-directed reach and specify the velocity and timing of the reach. The "control" phase of the movement determines the fine-tuning of certain elements of the reach, such as grip aperture, hand configuration, and target acquisition. We could therefore expect the reaches of optic ataxic patients to head generally in the right direction, under the influence of an efficient motor plan, but expect their final endpoint or grip formation to be inaccurate. This is not the typical observation with many optic ataxic patients, however, for whom the initial reach direction is also often grossly inaccurate. For example, nonfoveal patients (see Buxbaum \& Coslett [1997] for this distinction) typically misreach towards fixation when reaching to extrafoveal targets. It is important to note that, contrary to what Glover's model suggests, it is not the case that (a) their initial reach direction is accurate, and (b) their subsequent aberrant on-line control makes the reach inaccurate.

This is particularly apparent in patients with bilateral damage to the superior parietal lobes. Patients Mrs. D. (Carey et al. 1997), D.P. (Buxbaum \& Coslett 1997), J.J., and M.U. (Jackson et al. 2004) all present with bilateral superior parietal damage and all show a type of "magnetic misreaching" akin to nonfoveal optic ataxia. For example, Mrs. D., with asymmetric slowly progressive 